\title{
Society Bulletins
}

Visceral Medicine

Visc Med 2020;36:346-348

DOI: 10.1159/000510321

Pressestelle der Deutschen Krebsgesellschaft e. V.

Dr. Katrin Mugele

Kuno-Fischer-Str. 8, 14057 Berlin, Germany

Tel.: +49 30 3229329-60

mugele@krebsgesellschaft.de

\section{Professor Dr. Thomas Seufferlein ist neuer Präsident der Deutschen Krebsgesellschaft}

Die Mitglieder der Delegiertenversammlung der Deutschen Krebsgesellschaft (DKG) haben Professor Dr. Thomas Seufferlein zum Präsidenten der größten onkologischen Fachgesellschaft im deutschsprachigen Raum gewählt. Seufferlein löst Professor Dr. Olaf Ortmann vom Caritas-Krankenhaus St. Josef in Regensburg ab, der die DKG weiterhin als Vorstandsmitglied unterstützt. Darüber hinaus wählten die Delegierten der DKG Prof. Dr. Anja Mehnert-Theuerkauf vom Universitätsklinikum Leipzig, Prof. Dr. Ullrich Graeven von den Kliniken Maria Hilf GmbH Mönchengladbach und Prof. Dr. Frederik Wenz vom Universitätsklinikum Freiburg in den Vorstand der Fachgesellschaft.

Seufferlein ist Ärztlicher Direktor der Klinik für Innere Medizin I an der Uniklinik Ulm. Zu seinen Arbeitsschwerpunkten zählt die Internistische Onkologie und die Gastroenterologie. Neben seiner ärztlichen Tätigkeit engagiert er sich seit vielen Jahren in der DKG, unter anderem als Vizepräsident im Vorstand, als Vorsitzender der Zertifizierungskommission der DKG-zertifizierten Darmkrebszentren, als Sprecher der DKG im Lenkungsausschuss des Leitlinienprogramms Onkologie und als Mitglied der Leitgruppen „KolorektalesKarzinom” und,Pankreaskarzinom” in der Arbeitsgemeinschaft Internistische Onkologie.

„Gerade in der Krebsmedizin verzeichnen wir einen rasanten Zuwachs an medizinischen Erkenntnissen. Darauf müssen wir schnell reagieren, beispielsweise mit qualitativ hochwertigen onkologischen Leitlinien, die regelmäßig aktualisiert werden, und mit einem interdisziplinären Qualitätsmanagement, das eine kontinuierliche Verbesserung der Krebsversorgung ermöglicht. Für diese Ziele setzt sich die Deutsche Krebsgesellschaft seit vielen Jahren und sehr erfolgreich ein, nicht nur im Austausch mit Fachexperten und -expertinnen, sondern auch in der Diskussion mit Ent- scheidern aus Politik und Gesundheitswesen, unter anderem im Nationalen Krebsplan und der Nationalen Dekade gegen Krebs. Ich freue mich sehr, gemeinsam mit den anderen Vorstandsmitgliedern, allen Sektionen der DKG und unseren Partnern, der Deutschen Krebshilfe und dem Deutschen Krebsforschungszentrum, weiter an der Umsetzung dieser Ziele arbeiten zu dürfen", so Seufferlein.

Der Vorstand der DKG wird alle 2 Jahre gewählt und setzt sich nun wie folgt zusammen:

Präsident:

Prof. Dr. Thomas Seufferlein (Ulm)

Weitere Vorstandsmitglieder:

Prof. Dr. Olaf Ortmann (Regensburg), Prof. Dr. Anja

Mehnert-Theuerkauf (Leipzig), Prof. Dr. Ullrich

Graeven (Mönchengladbach), Prof. Dr. Frederik

Wenz (Freiburg)

Kongresspräsident des DKK 2022:

Prof. Dr. Michael Ghadimi (Göttingen)

Vorstandsmitglieder der DKG-Sektion A (repräsentieren die Landeskrebsgesellschaften):

Prof. Dr. Petra Feyer (Berlin)

Prof. Dr. Christian Jackisch (Offenbach)

Vorstandmitglieder der DKG-Sektion B (repräsentie-

ren die Arbeitsgemeinschaften und interdisziplinären

Studiengruppen der DKG):

Prof. Dr. Rainer Engers (Neuss) (Wahl bereits im

Vorjahr)

Prof. Dr. Andrea Tannapfel (Bochum)

Vorstandsmitglieder der DKG-Sektion C (fördernde

Mitglieder):

Dr. Michael Hanske (München)

PD Dr. Georg Isbary (Grenzach-Wyhlen)

DKG-Generalsekretär:

Dr. Johannes Bruns (Berlin) 


\section{Erfolgsgeschichte der Darmkrebsvorsorge jetzt fortsetzen - DGVS mahnt Rückkehr zur regulären Früherkennung an}

Um für die Corona-Pandemie gewappnet zu sein, wurden in den vergangenen Wochen weite Bereiche der Medizin umorganisiert: Kapazitäten für Intensivbetten wurden erhöht, planbare Operationen verschoben, Risikopatienten wurde geraten, nicht notwendige Arztbesuche abzusagen. Aktuell kehren Kliniken und Praxen schrittweise wieder zur Normalität zurück. Die Deutsche Gesellschaft für Gastroenterologie, Verdauungs- und Stoffwechselkrankheiten (DGVS) empfiehlt Patienten, nicht nur bei akuten Beschwerden ihren Arzt aufzusuchen, sondern jetzt auch die wichtigen Früherkennungsuntersuchungen, etwa die Vorsorgekoloskopie, wieder wahrzunehmen. Sonst drohe das Risiko, dass Krebserkrankungen zu spät erkannt würden. Diese Untersuchungen wurden in den vergangenen Wochen in großem Umfang abgesagt.

Vor allem endoskopische Eingriffe - zu denen beispielsweise Magen- und Darmspiegelungen zur Abklärung akuter Beschwerden wie Oberbauchschmerzen oder Schluckbeschwerden, aber auch Darmspiegelungen zur Darmkrebsfrüherkennung gehören - sind in den vergangenen Wochen in großem Umfang ausgefallen. „Unseren Schätzungen nach sind allein bei den Koloskopien, also den Darmspiegelungen, seit Auftreten der Coronakrise mindestens 20000 Untersuchungen in Deutschland entfallen", sagt Professor Dr. med. Heiner Wedemeyer, Mediensprecher der DGVS und Direktor der Klinik für Gastroenterologie, Hepatologie und Endokrinologie an der Medizinischen Hochschule Hannover. Sie wurden entweder seitens der Kliniken verschoben, oft jedoch auch von Patienten abgesagt, weil diese sich vor einer Ansteckung mit SARS CoV-2 in Kliniken oder Praxen fürchten.

„Gastroenterologische Abteilungen und Praxen setzen umfassende Hygienemaßnahmen um, um sowohl ihre Patienten als auch die Mitarbeiter vor einer Infektion zu schützen“, sagt Dr. med. Peter Buggisch, Leitender Arzt des Leberzentrums Hamburg und Vorstands- mitglied der DGVS. „Patienten können darauf vertrauen, dass sie bei uns sicher versorgt werden."

Vor allem akute Beschwerden - etwa starke Oberbauchschmerzen, Blut im Stuhl, blutiges Erbrechen oder plötzliche Gewichtsabnahme - sollten Betroffene zeitnah beim Gastroenterologen abklären lassen, sonst drohen ernsthafte gesundheitliche Probleme.

Aber auch bei den Früherkennungsuntersuchungen mahnen die Experten die Rückkehr zu regulären $\mathrm{Ab}$ läufen an. „Für einen gewissen Zeitraum ist das Verschieben oder Aussetzen von Vorsorge- und Früherkennungsmaßnahmen angesichts einer Ausnahmesituation vertretbar, aber längerfristig droht ein Anstieg zu spät erkannter Krebsfälle“, warnt Professor Dr. med. Helmut Messmann, Direktor der III. Medizinischen Klinik mit Schwerpunkt Gastroenterologie am Universitätsklinikum Augsburg und Sekretär der Sektion Endoskopie der DGVS. „Patienten sollten deshalb mit ihrem Arzt besprechen, ob ihre geplanten Vorsorgeuntersuchungen unmittelbar oder in der nahen Zukunft stattfinden können - und diese nicht etwa absagen oder in die ferne Zukunft verschieben." Dr. Ulrich Rosien, Leitender Arzt am Israelitischen Krankenhaus, Viszeral-Medizinisches Zentrum in Hamburg, und Vorsitzender der Sektion Endoskopie der DGVS, weist auf die unterschiedliche regionale Häufung von Covid-19-Infektionen in Deutschland hin. „Vorsorgekoloskopien sind unter den etablierten Schutzmaßnahmen sicher durchführbar. Mancherorts sind in der Pandemie-Situation die Kapazitäten noch reduziert; gerade in den Praxen und Ambulanzen ist das Angebot an endoskopischen Untersuchungen in der Regel aber nicht mehr eingeschränkt."

Das von den gesetzlichen Krankenkassen bezahlte Angebot zur Darmkrebsfrüherkennung gehört zu den effektivsten Früherkennungsmaßnahmen, die in der Medizin zu Verfügung stehen: In den ersten 10 Jahren nach Einführung der gesetzliche Darmkrebsfrüherken- 
nung sank die Darmkrebssterblichkeit bei Männern ab 55 Jahren um fast 21\%, bei Frauen dieser Altersgruppe sogar um mehr als 26\%. Ab dem Alter von 50 Jahren können Männer und Frauen mit dem immunologischen Stuhltest (iFOBT) jährlich ihren Stuhl auf verstecktes
Blut untersuchen lassen. Zudem bietet das Früherkennungsprogramm Männern ab 50 Jahren und Frauen ab 55 Jahren eine Darmspiegelung an, die zuverlässigste Methode der Früherkennung. Sie sollte alle 10 Jahre wiederholt werden. 